\title{
Calculation of Displacement Matrix Elements for Morse Oscillators
}

\author{
Zimei Rong, Dominique Cavagnat, and Laure Lespade \\ Laboratoire de Physico-Chimie Moléculaire, UMR 5803 CNRS \\ Université de Bordeaux I, \\ 351 cours de la Libération, F-33405 Talence Cedex, France \\ zimeirong@hotmail.com
}

\begin{abstract}
Displacement matrix elements of the Morse oscillators are widely used in physical and chemical computation. Many papers have been published about the analytical expressions of the displacement matrix elements. People still use an integration method rather than the analytical expressions to calculate the displacement matrix elements. Analytical expressions of the diagonal and off diagonal displacement matrix elements $\left\langle v+m\left|q^{n}\right| m\right\rangle$ with intermediate variables are derived, where $v, m$ and $n$ are non-negative integers and $n \bullet 6$. Calculation with these expressions is superior to that with numerical integration in precision, calculation speed and convenience.
\end{abstract}

\section{Introduction}

The Morse potential is the most popular anharmonic diatomic potential model in molecular spectroscopy and molecular dynamics. The matrix elements are widely used in calculation of transition energies, intensities and relative physical observables. Many physical quantities, such as dipole moment function, are functions of displacement, which gives to displacement matrix elements (abbreviated as matrix elements thereafter) extra importance. The Morse potential is expressed as [1]

$$
V(q)=D\left(1-e^{-a q}\right)^{2},
$$

where $D$ is the dissociation energy and $a$ is the scaling factor. The first advantage of the Morse oscillator is that its simple energy level equation represents very well the actual molecular energy levels and its parameters can easily be found from observed transition energies. Another advantage is the relatively simple expressions of its analytical wave functions, from which the analytical matrix elements are obtained [2 - 9]. Many previous papers have dealt with the analytical expressions of the Morse matrix elements. Heaps and Herzberg derived the analytical matrix elements for the $n=1$ and 2 [2]. Herman and Rubin obtained general expressions for the matrix elements [3]. However, these expressions have never gain broad acceptance because of their cumbersome formulation [10]. The algorithm of the analytical expressions is poor [8]. The analytical expressions involve computational difficulties of significant rounding 
off errors due to near cancellation terms in the summations $[3,5,7]$. Too much calculation is required for only some matrix elements of analytical expressions $[5,6,8]$. Only a few expressions for the diagonal $[4,5,7]$ and off diagonal matrix elements $[4$, 8] among the published analytical matrix elements are more convenient than an integral method. Thus, a numerical integration method was thought more convenient than the analytical expressions to calculate the matrix elements [11]. On the other hand, accuracy of the numerical integration will deteriorate with increase of $m, n$ and $v$ [6]. We have previously derived compact expressions of the matrix elements $\left\langle v\left|q^{n}\right| 0\right\rangle$ for $n$ - 6 without summations [12] and used in a simulation program of $\mathrm{CH}$ stretching methyl internal rotation [13]. But, in that paper, those expressions are limited to the calculation with the ground state.

In this paper, we derive general expressions for the diagonal and off diagonal matrix elements $\left\langle v+m\left|q^{n}\right| m\right\rangle$ for $n \bullet 6$, which are used in our spectroscopic calculations. To keep the expressions compact and calculation fast, intermediate variables are introduced. For verification, these analytical matrix elements are compared with those from numerical integrations of the analytical wave functions.

\section{Morse Oscillator}

The Schrödinger equation associated to the Morse oscillator is given by [1]

$$
-\frac{\hbar^{2}}{2 \mu} \frac{d^{2} \Psi(q)}{d q^{2}}+D\left(1-e^{-a q}\right)^{2} \Psi(q)=E \Psi(q) .
$$

The vibrational energy levels are given by [1]

$$
E_{v}=(v+1 / 2) \hbar \omega-(v+1 / 2)^{2} \hbar x,
$$

where $v$ is the quantum number, $\omega$ is the frequency and $x$ is the anharmonicity, which can be expressed with the parameters $D$ and $a$. Either the parameter pair $(\omega, x)$ or $(D$, a) uniquely determines a Morse oscillator. The frequency and anharmonicity are usually obtained from the observed transitions fitted to the Birge-Sponer equation,

$$
v_{v \leftarrow 0} / v=(\omega+x)+v x .
$$

Three intermediate parameters, $k=\omega / x, \beta=k-2 v-1$ and $y=k \exp (-a q)$ are used in the wave function formulation. The orthonomal wave functions are nondegenerate and are given by [1]

$$
|v\rangle=N_{v} e^{-y / 2} y^{\beta / 2} L_{v}^{\beta}(y),
$$

where the normalisation constant is given by

$$
N_{v}=[a \beta \Gamma(v+1) / \Gamma(k-v)]^{1 / 2},
$$


where $\bullet$ is the gamma function. In the solution, a generalised Laguerre polynomial is used, which is defined as [14]

$$
L_{v}^{\beta}(y)=\sum_{i=0}^{v} \frac{(-1)^{i} \Gamma(\beta+v+1)}{i !(v-i) ! \Gamma(\beta+i+1)} y^{i} .
$$

Generalised Laguerre polynomials can be calculated from the following recurrences [14]

$$
\begin{gathered}
L_{0}^{\beta}(y)=1, \\
L_{1}^{\beta}(y)=1+\beta-y, \\
v L_{v}^{\beta}(y)=(2 v-1+\beta-y) L_{v-1}^{\beta}(y)-(v-1+\beta) L_{v-2}^{\beta}(y) .
\end{gathered}
$$

\section{Displacement Matrix Elements}

The Morse matrix elements $\left\langle v+m\left|q^{n}\right| m>\right.$ can be calculated with numerical integrations of the wave functions in Eq. (5) or with the analytical expressions [see ref 2 - 9]. The deriving procedure of the analytical matrix elements can be divided into two parts [4]. The matrix elements of $y^{\lambda}$ are calculated first and then, the matrix elements of $q^{n}$ are calculated with the relationship:

$$
q^{n}=\left.\left(\frac{-1}{a}\right)^{n} \frac{d^{n}}{d \lambda^{n}}\left(\frac{y}{k}\right)^{\lambda}\right|_{\lambda=0} .
$$

The matrix elements of $y^{\lambda}$ were given as:

$<v+m\left|y^{\lambda}\right| m>=\frac{N_{0} N_{v}}{a} \sum_{i=0}^{m} \frac{\Gamma(k+\lambda-m-v-i-1) \Gamma(i-\lambda+1) \Gamma(i-\lambda+v+1)}{\Gamma(i+v+1) \Gamma(m-i+1) \Gamma(i+1) \Gamma(1-\lambda+v) \Gamma(1-\lambda-v)}$.

In evaluating the off diagonal matrix elements $\left\langle v+m\left|q^{n}\right| m\right\rangle$, where $v>0$, both gamma functions $\Gamma(1-\lambda-v)$ and polygamma functions $\psi^{(i)}(1-\lambda-v)$ are used, where $i$ is a non negative integer. They have poles at $\bullet=0$, which can be solved with the following two equations:

$$
\begin{aligned}
& \psi(1-\lambda-v) /\left.\Gamma(1-\lambda-v)\right|_{\lambda \rightarrow 0}=(-1)^{v} \Gamma(v), \\
& \psi(1-\lambda-v)=\psi(\lambda+v)+\pi \cot [(\lambda+v) \pi] .
\end{aligned}
$$


The matrix elements of $q^{n}$ are calculated by combining Eqs. (11) and (12). The number of terms in the matrix elements increases dramatically as $n$ increases. To keep the expressions compact, the following intermediate variables are introduced

$$
\begin{gathered}
y_{0}=\frac{(-1)^{v}}{v} \sqrt{\frac{(k-2 m-1)(k-2 m-2 v-1) \Gamma(m+1) \Gamma(m+v+1)}{\Gamma(k-m) \Gamma(k-m-v)}}, \\
y_{c}=\Gamma(k-m-v-i-1) / \Gamma(m-i+1), \\
y_{i+1}=\psi^{(i)}(k-m-v-i-1)-\Gamma(i+1)\left[i^{-i-1}+\sum_{j=v+1}^{j=v+i} j^{-i-1}\right]-\delta_{i 0} \ln k .
\end{gathered}
$$

The off diagonal matrix elements can then be expressed as

$$
\begin{gathered}
<v+m|q| m>=-y_{0} a^{-1} \Gamma(k-m-v) /(k-2 m-v-1) \Gamma(m+1), \\
<v+m\left|q^{2}\right| m>=y_{0} a^{-2} \sum_{i=0}^{m} y_{c}\left(2 y_{1}\right), \\
<v+m\left|q^{3}\right| m>=-y_{0} a^{-3} \sum_{i=0}^{m} y_{c}\left(3 y_{1}^{2}+3 y_{2}-\pi^{2}\right), \\
<v+m\left|q^{4}\right| m>=y_{0} a^{-4} \sum_{i=0}^{m} y_{c}\left(4 y_{1}^{3}+12 y_{1} y_{2}+4 y_{3}-4 y_{1} \pi^{2}\right), \\
<v+m\left|q^{5}\right| m>=-y_{0} a^{-5} \sum_{i=0}^{m} y_{c}\left(5 y_{1}^{4}+30 y_{1}^{2} y_{2}+20 y_{1} y_{3}+15 y_{2}^{2}+5 y_{4},\right. \\
\left.+10 y_{1}^{2} \pi^{2}+10 y_{2} \pi^{2}-\pi^{4}\right) \\
<v+m\left|q^{6}\right| m>=y_{0} a^{-6} \sum_{i=0}^{m} y_{c}\left(60 y_{1}^{3} y_{2}+60 y_{1}^{2} y_{3}+90 y_{1} y_{2}^{2}+30 y_{1} y_{4}+60 y_{2} y_{3}\right. \\
\left.+6 y_{1}^{5}+6 y_{5}-20 y_{1}^{3} \pi^{2}-60 y_{1} y_{2} \pi^{2}-20 y_{3} \pi^{2}+6 y_{1} \pi^{4}\right)
\end{gathered}
$$

For the diagonal matrix elements the following intermediate variables are used,

$$
\begin{gathered}
x_{d}=(k-2 m-1) \Gamma(m+1) / \Gamma(k-m), \\
x_{c}=\Gamma(k-m-i-1) / \Gamma(m-i+1), \\
x_{i}=\psi^{(i)}(k-m-i-1)-2 \Gamma(i+1) \sum_{j=1}^{i} j^{-i-1}-\delta_{i 0} \ln (k) .
\end{gathered}
$$


The diagonal matrix elements $<m\left|q^{n}\right| m>$ can thus be written as

$$
\begin{gathered}
<m|q| m>=-x_{d} a^{-1} \sum_{i=0}^{m} x_{c} x_{0}, \\
<m\left|q^{2}\right| m>=x_{d} a^{-2} \sum_{i=0}^{m} x_{c}\left(x_{0}^{2}+x_{1}\right), \\
<m\left|q^{3}\right| m>=-x_{d} a^{-3} \sum_{i=0}^{m} x_{c}\left(x_{0}^{3}+3 x_{0} x_{1}+x_{2}\right), \\
<m\left|q^{4}\right| m>=x_{d} a^{-4} \sum_{i=0}^{m} x_{c}\left(x_{0}^{4}+6 x_{0}^{2} x_{1}+4 x_{0} x_{2}+3 x_{1}^{2}+x_{3}\right), \\
<m\left|q^{5}\right| m>=-x_{d} a^{-5} \sum_{i=0}^{m} x_{c}\left(x_{0}^{5}+10 x_{0}^{3} x_{1}+10 x_{0}^{2} x_{2}+15 x_{0} x_{1}^{2}+5 x_{0} x_{3}\right. \\
\left.+10 x_{1} x_{2}+x_{4}\right) \\
<m\left|q^{6}\right| m>=x_{d} a^{-6} \sum_{i=0}^{m} x_{c}\left(x_{0}^{6}+15 x_{0}^{4} x_{1}+20 x_{0}^{3} x_{2}+45 x_{0}^{2} x_{1}^{2}+15 x_{0}^{2} x_{3}\right. \\
\left.+60 x_{0} x_{1} x_{2}+6 x_{0} x_{4}+15 x_{1}^{3}+15 x_{1} x_{3}+10 x_{2}^{2}+x_{5}\right)
\end{gathered} .
$$

The matrix elements with the ground state $<v\left|q^{n}\right| 0>$ were derived previously [12] and are listed for comparison. With the following intermediate variables

$$
\begin{gathered}
y_{0}=(-1)^{v} \sqrt{(k-2 v-1) \Gamma(v) \Gamma(k-v-1) / v(k-v-1) \Gamma(k-1)}, \\
y_{i+1}=\psi^{(i)}(k-v-1)+\psi^{(i)}(v)-(-1)^{(i)} \psi^{(i)}(1)-\delta_{i 0} \ln k,
\end{gathered}
$$

the off diagonal matrix elements are expressed as

$$
\begin{gathered}
<v|q| 0>=-y_{0} a^{-1}, \\
<v\left|q^{2}\right| 0>=y_{0} a^{-2}\left(2 y_{1}\right), \\
<v\left|q^{3}\right| 0>=-y_{0} a^{-3}\left(3 y_{1}^{2}+3 y_{2}-\pi^{2}\right), \\
<v\left|q^{4}\right| 0>=y_{0} a^{-4}\left(4 y_{1}^{3}+12 y_{1} y_{2}+4 y_{3}-4 y_{1} \pi^{2}\right), \\
<v\left|q^{5}\right| 0>=-y_{0} a^{-5}\left(5 y_{1}^{4}+30 y_{1}^{2} y_{2}+20 y_{1} y_{3}+15 y_{2}^{2}+5 y_{4}\right. \\
\left.+10 y_{1}^{2} \pi^{2}+10 y_{2} \pi^{2}-\pi^{4}\right)
\end{gathered}
$$




$$
\begin{aligned}
<v\left|q^{6}\right| 0> & =y_{0} a^{-6}\left(60 y_{1}^{3} y_{2}+60 y_{1}^{2} y_{3}+90 y_{1} y_{2}^{2}+30 y_{1} y_{4}+60 y_{2} y_{3}\right. \\
& \left.+6 y_{1}^{5}+6 y_{5}-20 y_{1}^{3} \pi^{2}-60 y_{1} y_{2} \pi^{2}-20 y_{3} \pi^{2}+6 y_{1} \pi^{4}\right)
\end{aligned}
$$

The diagonal matrix elements are expressed with intermediate variables

$$
x_{i}=\psi^{(i)}(k-1)-\delta_{i 0} \ln (k),
$$

as

$$
\begin{gathered}
<0|q| 0>=-a^{-1} x_{0}, \\
<0\left|q^{2}\right| 0>=a^{-2}\left(x_{0}^{2}+x_{1}\right) \\
<0\left|q^{3}\right| 0>=-a^{-3}\left(x_{0}^{3}+3 x_{0} x_{1}+x_{2}\right), \\
<0\left|q^{4}\right| 0>=a^{-4}\left(x_{0}^{4}+6 x_{0}^{2} x_{1}+4 x_{0} x_{2}+3 x_{1}^{2}+x_{3}\right), \\
<0\left|q^{5}\right| 0>=-a^{-5}\left(x_{0}^{5}+10 x_{0}^{3} x_{1}+10 x_{0}^{2} x_{2}+15 x_{0} x_{1}^{2}+5 x_{0} x_{3}+10 x_{1} x_{2}+x_{4}\right), \\
<0\left|q^{6}\right| 0>=a^{-6}\left(x_{0}^{6}+15 x_{0}^{4} x_{1}+20 x_{0}^{3} x_{2}+45 x_{0}^{2} x_{1}^{2}+15 x_{0}^{2} x_{3}\right. \\
\left.+60 x_{0} x_{1} x_{2}+6 x_{0} x_{4}+15 x_{1}^{3}+15 x_{1} x_{3}+10 x_{2}^{2}+x_{5}\right)
\end{gathered}
$$

The general expressions of the off diagonal matrix elements $<v+m\left|q^{n}\right| m>$ in Eqs. (18) - (23) and the matrix elements $\left\langle v\left|q^{n}\right| 0>\right.$ in Eqs. (35) - (40) have the same coefficients in the corresponding expressions, the summation only appearing in the general expressions. The same pattern occurs by comparing the diagonal matrix elements $<m\left|q^{n}\right| m>$ in Eqs. (27) - (32) with the matrix elements $<0\left|q^{n}\right| 0>$ in Eqs. (42) - (47). The $n$ is limited to 6 in our work, and can be extended further simply, however the term of the expression increases with the $n$ increasing. The matrix elements $<v+m\left|q^{n}\right| m>$ are verified by numerical comparison with those from the analytical matrix elements $<v\left|q^{n}\right| 0>$ and those from numerical integration. For example, the same result $\left\langle 6\left|q^{6}\right| 0\right\rangle=0.00000123459177 \AA^{6}$ is obtained from the Eqs. (23), (40) and numerical integration (with reduced mass $0.97959254204827 \mathrm{amu}$, frequency 2988.84 $\mathrm{cm}^{-1}$ and anharmonicity $51.59 \mathrm{~cm}^{-1}$ ). However, the calculation with the numerical integration needs much more time than that with the analytical expressions. High accuracy is achieved in our expressions without the sign alternation in the summation, which causes the round off errors in previous expressions $[4,5,7]$.

\section{Conclusion}

The analytical expressions of the Morse matrix elements $<v+m\left|q^{n}\right| m>$ for $n \bullet 6$ have been derived and programmed with the input of the reduced mass, frequency and 
anharmonicity. Intermediate variables are employed to keep the expressions compact and computation fast. Our expressions are exact and verified with a numerical integration method. Compared with the previous analytical matrix elements, great accuracy, simplicity and fast computation are achieved with these expressions.

\section{References}

1. Morse, P. M.: Phys. Rev. 34, (1929) 57-64

2. Heaps, H. S., Herzberg, G.: Z. Phys. 133, (1952) 48-64

3. Herman, R., Rubin, R. J.: Astrophys. J. 121, (1955) 533-540

4. Sage, M. L.: Chem. Phys. 35, (1978) 375-380

5. Gallas, J. A. C.: Phys. Rev. A 21, (1980) 1829-1834

6. Sovkov, V. B., Ivanov, V. S.: Opt. Spectrosc. 59, (1986) 733-735

7. Requena, A., Piñeiro, A. L., Moreno, B.: Phys. Rev. A 34, (1986) 4380-4386

8. Nagaoka, M., Yamabe, T.: Phys. Rev. A. 38, (1988) 5408-5411

9. López-Piñeiro, A., Moreno, B.: Phys. Rev. A 41, (1990) 1444-1449

10. Ogilvie, J. F.: The Vibrational and Rotational Spectroscopy of Diatomic Molecules. Academic Press, San Diego, (1998)

11. Truhlar, D. G., Onda, K.: Phys. Rev. A 23, (1981) 973-974

12. Rong, Z.: Ph.D. Thesis, Otago University, (2002)

13. Rong Z., Kjaergaard, H. G.: J. Phys. Chem. A 106, (2002) 6242-6253

14. Abramowitz; M., Stegun, I. A.: Handbook of Mathematical Functions. Dover, New York, (1965).

15. Herzberg, G.: Spectra of Diatomic Molecules. D. Van Nostrand Company, Inc., New York, (1951), 501-581

\section{Appendix: Polygamma Function}

The polygamma functions are extensively used in the expressions. The asymptotic formula is given as follows [14]

$$
\psi^{(i)}(z)=(-1)^{(i-1)}\left(\frac{\Gamma(i)}{z^{i}}+\frac{\Gamma(i+1)}{2 z^{i+1}}+\sum_{j=1}^{\infty} B_{2 j} \frac{\Gamma(2 j+i)}{\Gamma(2 j+1) z^{2 j+i}}\right) .
$$

where $B_{2 j}$ are the Bernoulli numbers. For most molecules [15], $k$ value or the argument $z$ is large enough, so that only very few terms are required to gain a high accuracy. 